\title{
Smart energy management system for utility source and photovoltaic power system using FPGA and ZigBee
}

\author{
Yousaf H. Khattak ${ }^{1}$, Tahir Mahmood ${ }^{1}$, Khurshid Alam ${ }^{1}$, Tahir Sarwar ${ }^{2}$, Inayat Ullah ${ }^{2}$, \\ Hanif Ullah ${ }^{3}$ \\ ${ }^{1}$ Department of Electrical Engineering, University of Engineering \& Technology, Taxila, Pakistan \\ ${ }^{2}$ Department of Electrical Engineering, Federal Urdu University of Arts, Science \& Technology, Islamabad, Pakistan \\ ${ }^{3}$ Polytechnic University of Valencia, Spain
}

\section{Email address:}

yousaf_hameed@engineer.com (Y. H. Khattak), tahir.mehmood@uettaxila.edu.pk (T. Mahmood), khurshiid.alam@gmail.com (K. Alam), tahir.sarwar89@gmail.com (T. Sarwar), inayatmz@gmail.com (I. Ullah),hanifuet@yahoo.com (H. Ullah)

\section{To cite this article:}

Yousaf H. Khattak, Tahir Mahmood, Khurshid Alam, Tahir Sarwar, Inayat Ullah, Hanif Ullah. Smart Energy Management System for Utility Source and Photovoltaic Power System Using FPGA and ZigBee. American Journal of Electrical Power and Energy Systems.

Vol. 3, No. 5, 2014, pp. 86-94. doi: 10.11648/j.epes.20140305.11

\begin{abstract}
Renewable energy resources are being used to overcome energy shortage. From the point of view of energy management the interconnectivity of electric utility with renewable energy resources is difficult. Renewable energy resources need to be managed with electric utility. A Smart Energy Management System (SEMS) is designed and developed for monitoring an efficient load management of electric utility and photovoltaic power system is presented in this research. The design consists of an Energy Management Center (EMC) and Field Programmable Gate Array (FPGA). Energy Management Center shows the runtime data and also keeps the data log and offers control of the load shifting between utility source and photovoltaic power system. Analog to digital converter is used to interface the current and voltage sensors with FPGA. ZigBee is connected for wireless radio data transmission between the FPGA and energy management \& monitoring center. The SEMS increases the efficiency of energy up to 10.5 percent in comparison with normal systems.
\end{abstract}

Keywords: FPGA, Energy Management, ZigBee, Renewable Energy, Smart Grid

\section{Introduction}

To meet the increasing demands of electrical energy new thermal power generation units are required to be installed. In thermal power plant the prime mover is driven by steam, water is first heated which turns into steam and spins a turbine which drives an electrical generator. This arrangement increases the cost of electricity and also not eco-friendly because it produces an adds a large part of $\mathrm{CO}_{2}$ emissions to the atmosphere. Integration of renewable energy resources with utility source are used as cost effective solution and also eco-friendly. They along with efficient energy management arrangements help to avoid the blackout caused by the conventional load shedding. $[1,2,3,5]$

For any country social economic development energy resources are the backbone. It is necessary for the production and contributes revenue in the budget. Energy is essential for the running of industry, transportation and lightning of the cities. The rapid increase in power demand because of industrial advancement, increase in population and improvement of living standards pose a serious challenge for power distribution systems.

In Pakistan energy crisis is growing because energy production is insufficient to meet the increasing energy demand of the country, currently our country is facing the critical energy problems and makes the situation even worse because of transmission failures due to obsolete facilities, stealing of electricity and shortage of hydropower. Therefore, utility companies follow the trend/method of load-shedding to avoid system failure or major breakdown where demand is greater than total generation. Further, tripping of feeders originating from a substation is also observed a common practice.

To overcome energy deficiency, different renewable energy resources are being used. They contribute an important role in the energy management solutions. 
Renewable energy resources are difficult to interconnect with main electric utility from the point of view of energy management. Renewable energy resources need to be managed with electric utility [1]. Energy management is a subject of great significance and complexity. It consists in selecting between a set of sources that are able to produce energy for loads by reducing costs and losses in distribution area. [2]

The variables that we need to take into account to improve efficiency of energy management system are timely power demands; solar cell generation depends on current battery storage status and weather conditions. These variables are not considered in the currently available solutions, so it is difficult to anticipate high efficiency of SEMS. Thus, for higher efficiency of a system, for proper energy management these methods need to be acquired/monitor.

In this research we proposed the smart energy management and monitoring system of photovoltaic power system and utility source based on FPGA and ZigBee. We design SEMS and implemented it on Spartan 3AN FPGA using Xilinx ISE tool and verify the performance as to how competently it manages energy using photovoltaic power system and utility source. For this purpose different techniques are implemented. As the technology has developed, SEMS has emerged as an effective tool for saving energy. This is important to increase the efficiency of system and to minimize the cost.

Verilog HDL coding and synthesis will be done in Xilinx ISE Tools. Model of FPGA will be finalized once the synthesis is complete and the number of combinational logic blocks and lookup tables used to implement the design will be finalized.

The main objectives of this research are to design and develop SEMS to increase the efficiency of a system.

- To design and implement efficient Smart Energy Management System algorithm on FPGA.

- To provide proper power management services and improve efficiency of SEMS.

- Architecture design of SEMS.

- Hardware Implementation of Energy Management System.

\section{Related Work}

Intelligent energy management system (IEMS) of utility source with photovoltaic power system is presented in [1]. IEMS is a part of a smart grid that can enable demand response applications for domestic customers. IEMS algorithm is developed for managing higher electrical load appliances. Domestic loads are managed by algorithm according to their precise priorities and guarantee the total consumption of electrical load appliances below definite levels. [2]

Energy Management is a method to decrease the power consumption of electronic system by selectively shut off the priority system. [3]

There are several studies and different commercial products including wind power and photovoltaic power in propagation of environment friendly technologies and they have significant roles. The power market would become more significant and the sustainable development of renewable energy as the influence of the smart grid is expected at wider scale. It is possible that the impact of renewable energy, sustainable growth will be much larger with the Smart Grid. In this type of generating systems, the interconnectivity of electric utility with renewable energy resources is difficult. Renewable energy resources need to be managed with electric utility. Moreover, renewable energy sources provide power which is more or less indefinitely, but this problem is expected to be stable. Therefore, for greater performance of renewable energy sources and to monitor these criteria, an intelligent system is needed. Also provides proper management services. [4]

In [5] SEMS is presented for effective electrical load management. It has two parts first is energy management center (EMC) containing graphical user interface (GUI). The runtime information is shown by EMC; it also keeps the data logging with electrical load control. Other part is load scheduling. For the illustration of results of load scheduling MATLAB simulations are used. Implemented hardware model is using human machine interface (HMI) and contains PIC18F4520 microcontroller and Zigbee.

Renewable energy systems such as wind power generation, fuel cells and photovoltaic power generation are getting a huge attention globally. As compare to other generating systems such as thermal and nuclear power plants, Renewable energy systems are eco-friendly because they are not emitting pollution like emission of a large amount of $\mathrm{CO}_{2}$ to the atmosphere when they generate electrical energy from thermal power plant and also do not discharge nuclear wastes as in nuclear power plants.

Renewable energy systems have a host of economic benefits, social benefits and environmental benefits. To solve several energy problems, renewable energy systems are considered as the important solution. They have also some disadvantages like steady output power can be obtained from thermal and nuclear plants but the renewable power generating plants such as wind mills and solar power generation do not provide steady power because it depends on the weather conditions, hourly variation and season[1].

At noon, maximum power can be achieved from photovoltaic power system and minimum power can be achieved from wind mills whereas, at night time no power can be achieved from photovoltaic power system and maximum power achieved from wind mills [1, 7] Due to non-steady power they also require energy storage system but because of higher costs they are not easy to install on large scale. [8]

In this paper, we have proposed SEMS which have emerged as an effective tool for saving energy. This is important to increase the efficiency of system and to minimize the cost. 


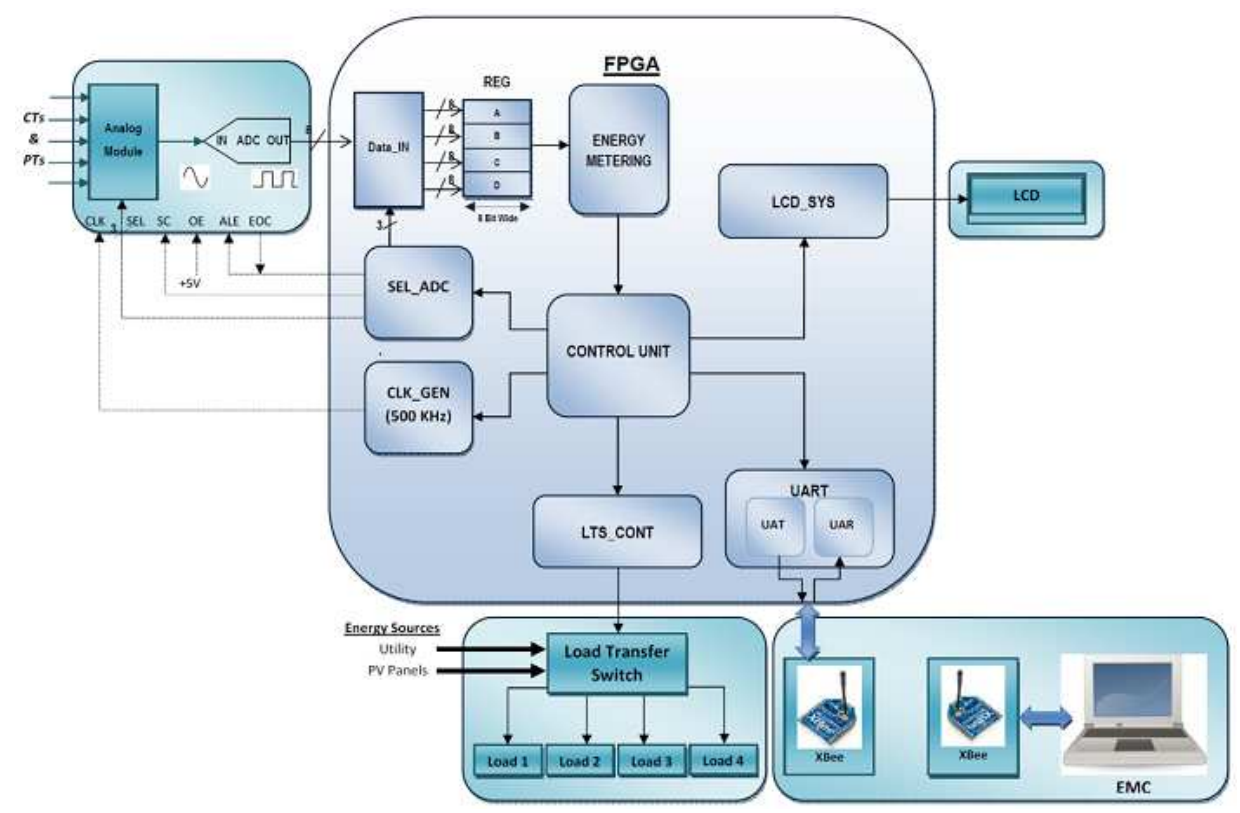

Figure 1. Hardware Architecture of Smart Energy Management System.

\section{System Architecture and Implementation}

Hardware Architecture of SEMS is shown in Figure1. It consists of the load transfer switches, analog to digital converter, ZigBee, current sensors, voltage sensors and control unit. The control unit part is situated in the middle. The SEMS offers the ability to sense the consumed power, transfer load to photovoltaic power system and utility source according to the usage and sends information through the ZigBee to EMC.

The system uses the CT and PT sensors to measure the consumed power. An analog value is measured by the CT and PT sensors and then converted to digital value that can be handled in the control unit by the energy metering block. The SEMS can determine consumption of power utilized by the loads. This power consumption value is then stored in the registers in control unit. If a monitoring value varies, SEMS transfer load to photovoltaic power system and utility source according to the usage.

The desired SEMS will be implemented using Xilinx ISE Tools and Spartan 3AN FPGA starter kit will be used as the hardware platforms. Verilog HDL coding and synthesis will be done in Xilinx ISE Tools. Model of FPGA will be finalized once the synthesis is complete and the number of combinational logic blocks and lookup tables used to implement the design. Microcontroller processing speed is less than FPGA because FPGAs are concurrent while microcontroller is always sequential. This makes FPGAs better suited for real-time applications. FPGA are flexible, we can add /subtract the functionality as required. Another advantage of using FPGA is to reduce the size of hardware.

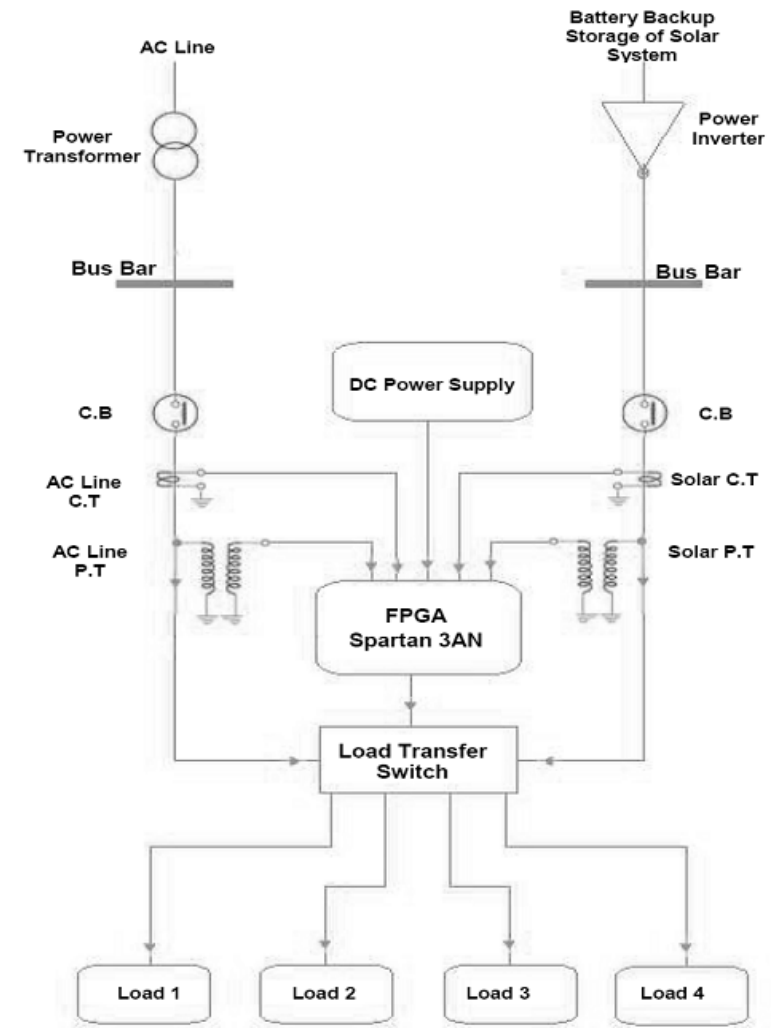

Figure 2. Single Line Diagram of SEMS.

Xilinx Spartan 3AN FPGA Starter kit is used as control unit, which is the brain of an SEMS. ADC 0809 provides input of physical quantities to control unit, which is taken from sensing part i.e. current transformers and potential transformers. FPGA receives information from $\mathrm{ADC}$ and stored it in temporary registers. For the working of ADC, control signals are also generated from the FPGA. These controls signals are Start Conversion (SC), Output Enable (OE), Address Latch 
Enable (ALE), End of Conversion (EOC), Clock of $500 \mathrm{KHz}$ (CLK) and Select (SEL) as declared in figure 2.

The stored information is processed by the Energy Metering block i.e. voltages, currents and powers of utility and PV system.

The data is then sent to the control unit. Main function of control unit is to control and generate all the control signals and $500 \mathrm{KHz}$ clock for the running of analog to digital converter. FPGA controls the load transfer switch and also sends the data to Energy Management Center (EMC) through universal asynchronous transmitter (UAT) block and Zigbee interface. FPGA also receives data from Energy Management Center through universal asynchronous receiver (UAR) block and Zigbee interface. Hardware specification of photovoltaic power system is stated in table 1

Table 1. Hardware Specification of Photovoltaic Power System.

\begin{tabular}{ll}
\hline Classification & Value \\
\hline Photovoltaic Panel $\left(\mathrm{P}_{\max }\right)(6)$ & $135 \mathrm{~W}(810 \mathrm{~W})$ \\
Inverter Rating $\left(\mathrm{P}_{\max }\right)$ & $4500 \mathrm{~W}$ \\
Battery Backup Storage Capacity $(4)$ & $220 \mathrm{Ah}(880 \mathrm{Ah})$ \\
Battery Voltage $\left(\mathrm{V}_{\text {out }}\right)$ & $12 \mathrm{~V}$ \\
Battery Power & $10560 \mathrm{Wh}$ \\
\hline
\end{tabular}

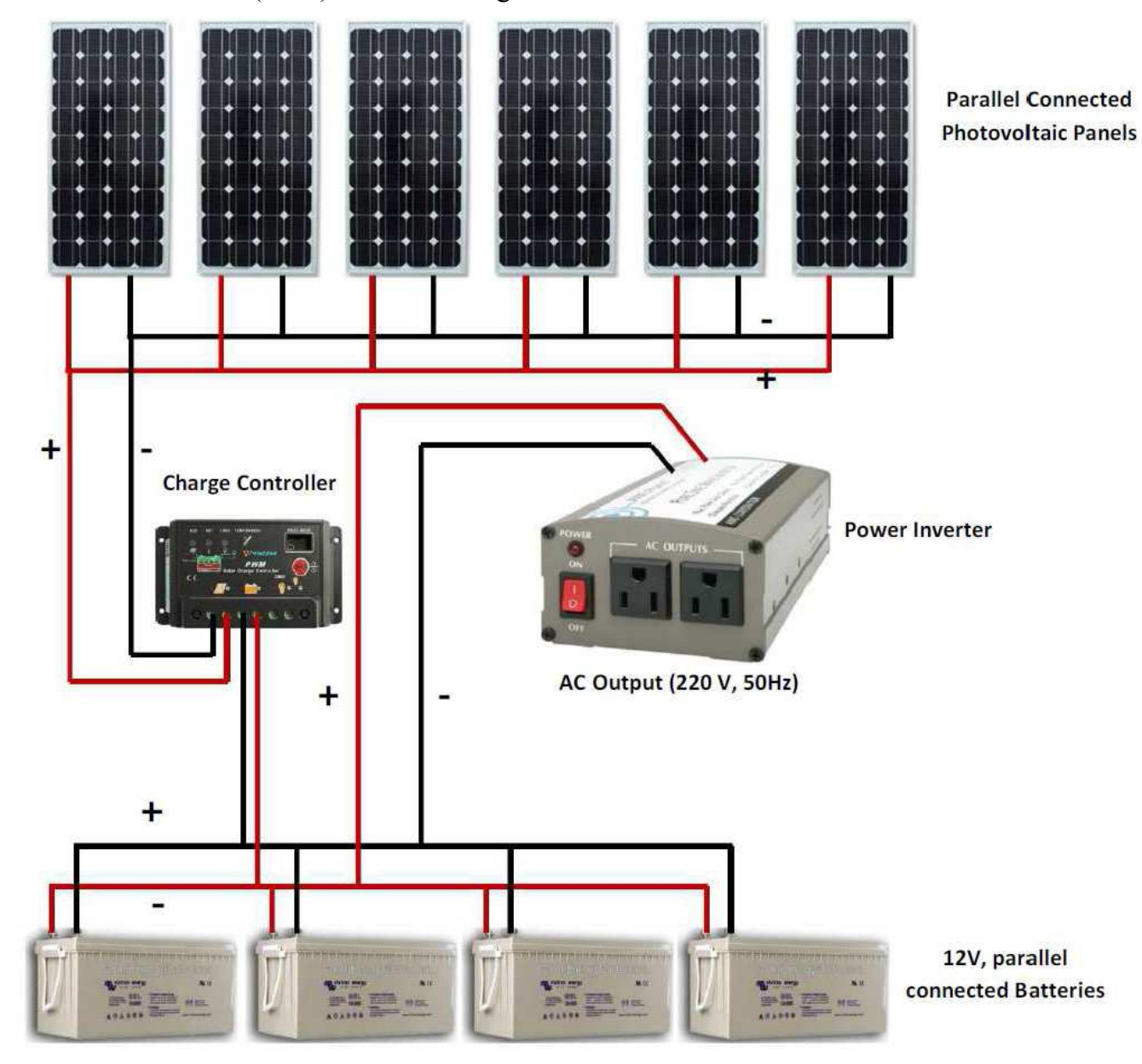

(A)

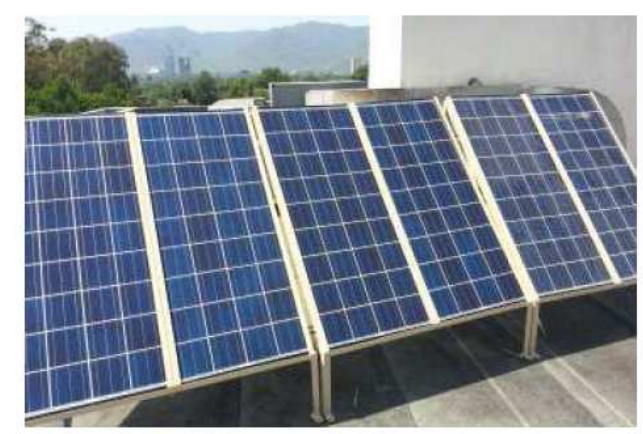

(B)

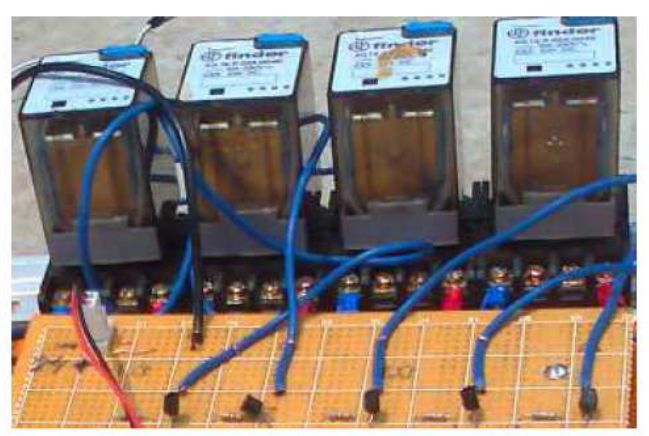

(C)

Figure 3. (A) Photovoltaic Power Generation System (B) 6 solar panels (C) Electrical Contactor Relays for Load Transfer. 


\subsection{Efficiency Based Technique}

The basic idea of the system based on efficiency is the efficiently working of the SEMS and emphases on the use of the photovoltaic system and utility sources. SEMS collects the information about the utility and the condition of battery status of photovoltaic power system and by the current transformer and potential transformer. This information is then sends to the EMC of SEMS via Zigbee. Here the power consumption is checked and compared with the energy sources.

The collected data by the sensors of photovoltaic power system and utility sources are transformed into specific factor (f).The SEMS decides whether it uses the power from photovoltaic power system or from utility source. Factor (f) is basically the difference of the available battery power of photovoltaic power system in watts and the expected power demand i.e. current power consumption in watts.
The system chooses to use the photovoltaic power system or utility source on the bases of following conditions.

$\mathrm{f}>$ Default Value: use power from the photovoltaic power system

$\mathrm{f}=$ Default Value: use power from both sources (utility source and photovoltaic power system)

$\mathrm{f}<$ Default Value: use only power from the utility source

If $\mathrm{f}$ is higher than default value, SEMS selects to use power from photovoltaic power system. If $f$ equals to the default value, SEMS selects to use power from both sources i.e. utility source and photovoltaic power system and turn off the photovoltaic power system power. When $\mathrm{f}$ is smaller, SEMS chooses to use the utility source. According to the batteries efficiency, default value is adjusted. Algorithm of efficiency based technique is shown in Figure 4.

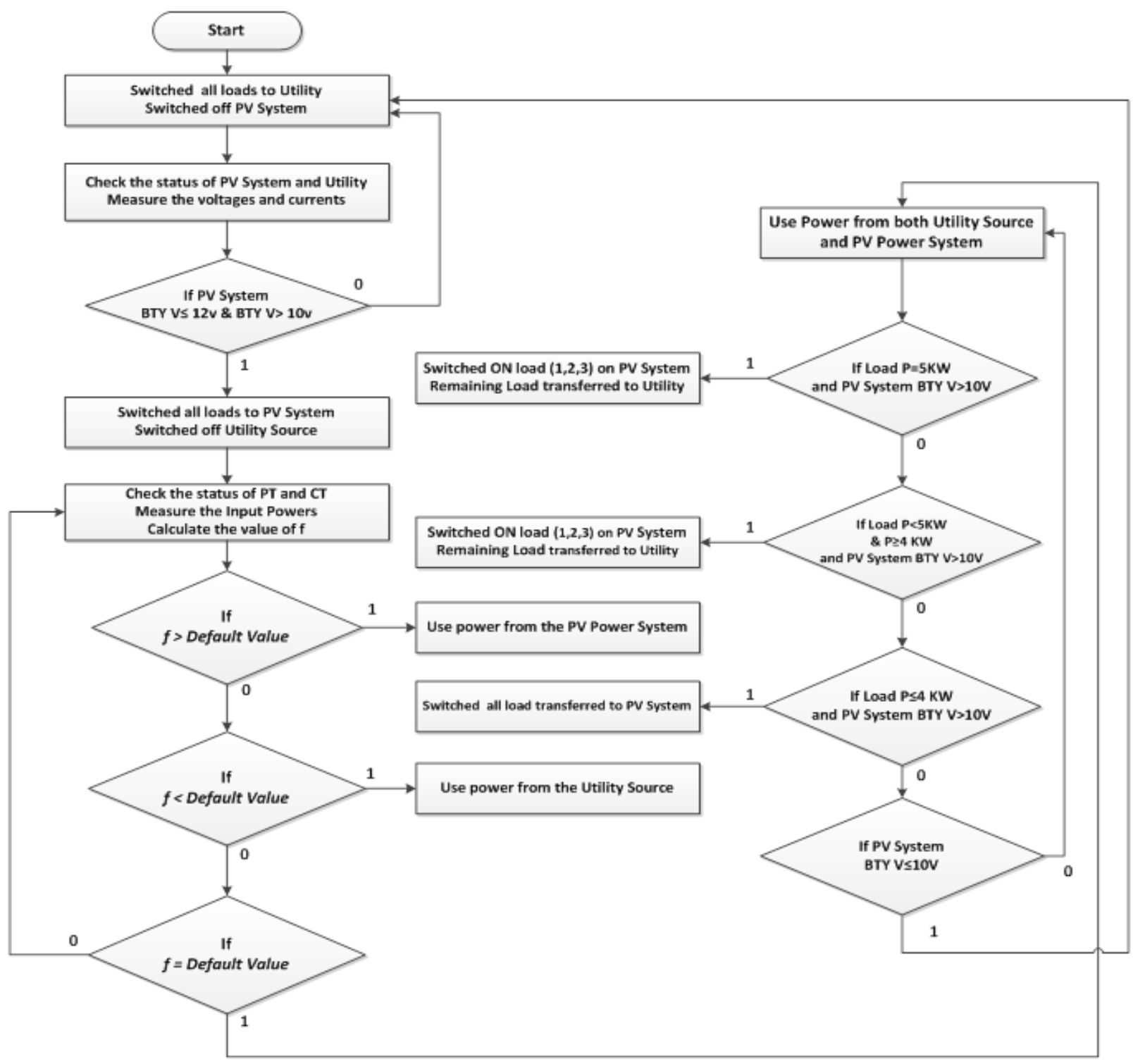

Figure 4. Algorithm of Efficiency Based Technique. 


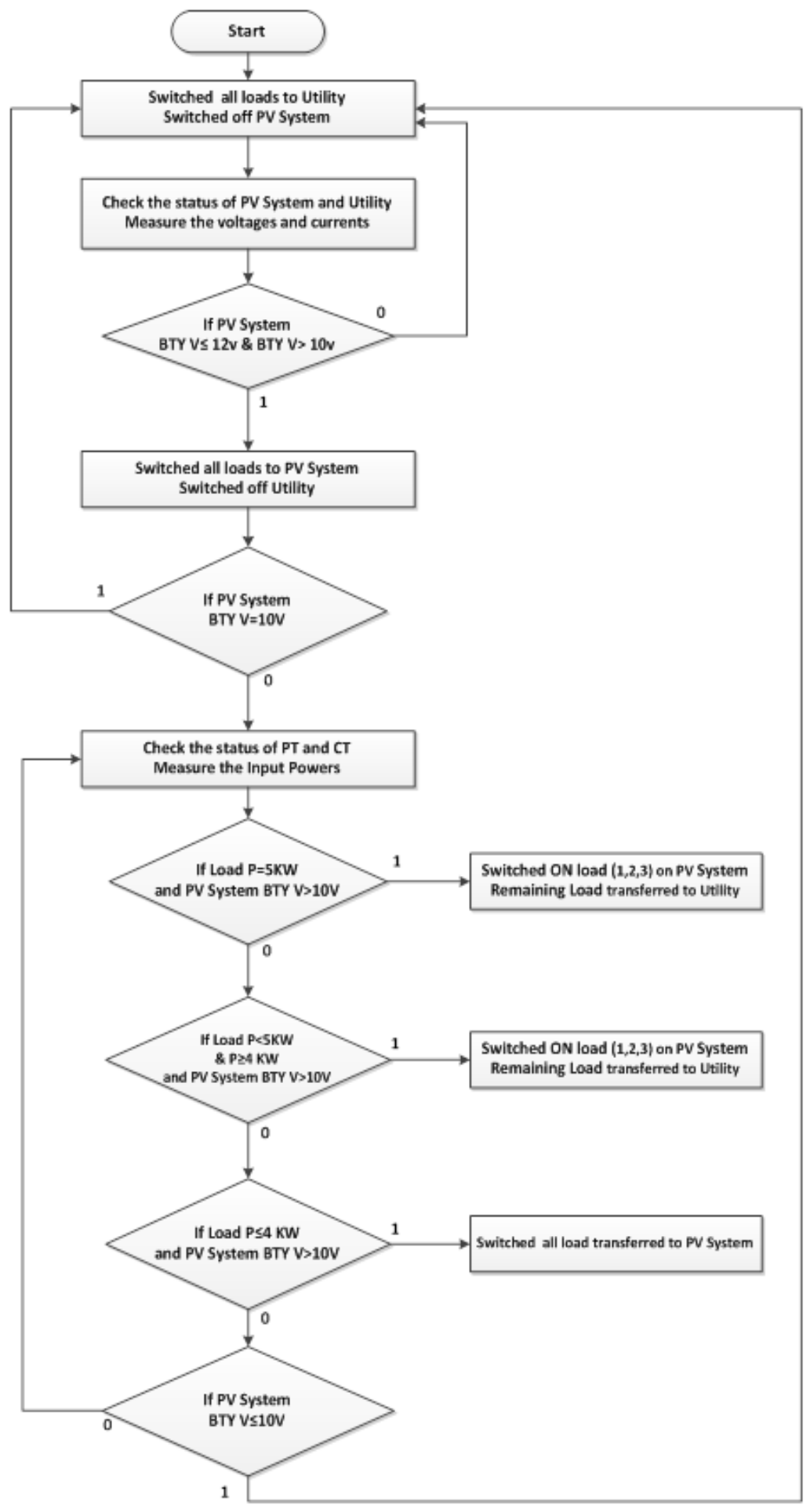

Figure 5. Algorithm of User Based Technique.

\subsection{User Based Technique}

User based technique is a simple method of the selection of energy source according to the requirement of electrical load. For user based technique EMC has a switch to select the working mode. Four switches are also available for managing load priorities. The SEMS controls the power except for loads which have priority. The user based technique manages the complete system for the ease of consumer's. It has a simpler algorithm as shown in Figure 5.

\subsection{Energy Management Center}

Energy Management Center (EMC) is basically simple and easy to use graphical user interface for monitoring and data logging of voltage and current of photovoltaic system and utility. It has voltmeter, ammeter and power meter for the 
indication and measurement of utility and also for photovoltaic power system. Voltage, current and power data's are shown on these meters. Data are updated on every second for data logging of voltage and currents in a table. It has three switches i.e. EOT, UOT and NLC used for the selection of efficiency based technique, user based technique and for normal load control scheme respectively. For normal load control scheme other four switches are also available for the transfer of loads between photovoltaic power system and utility source. The snap shots of EMC interface are shown in Figure 6 and Figure 7.

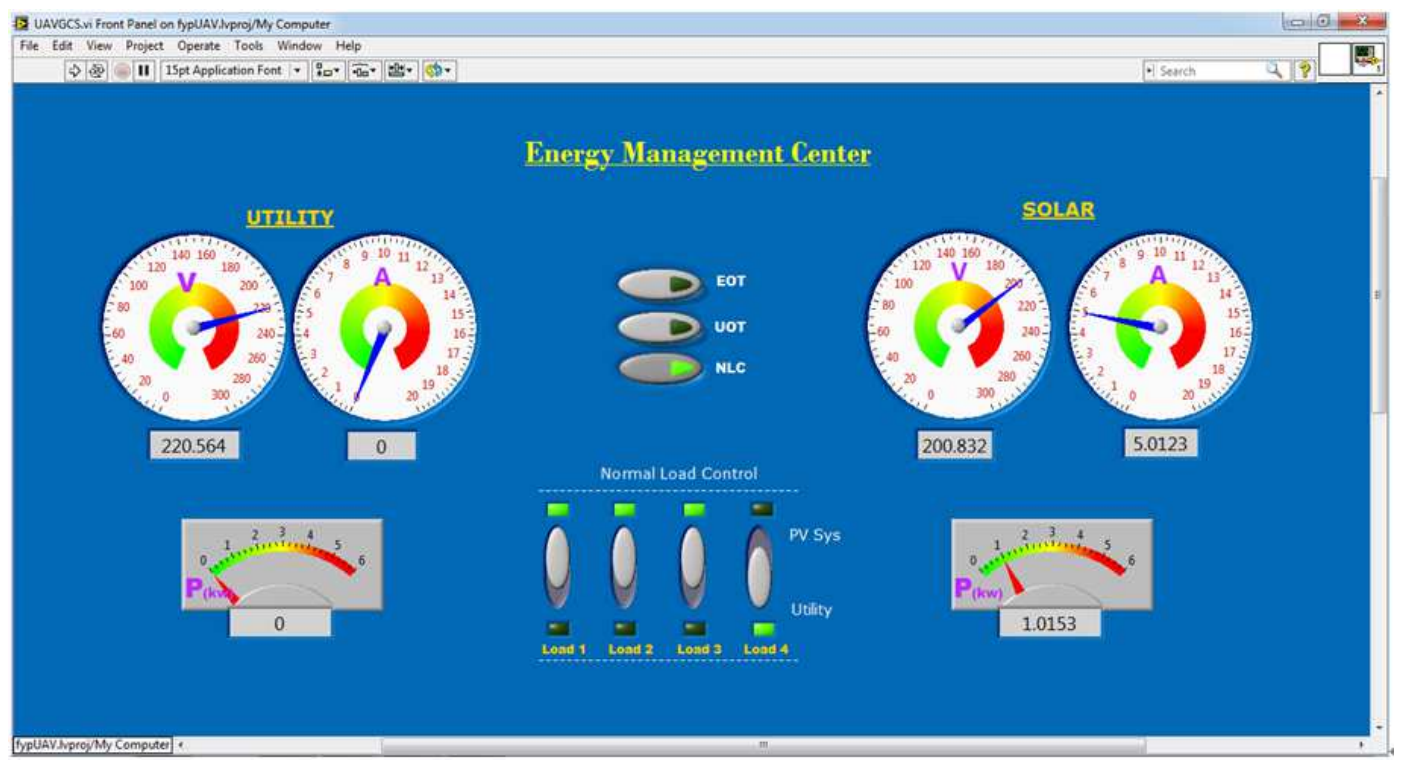

Figure 6. Main window of EMC.

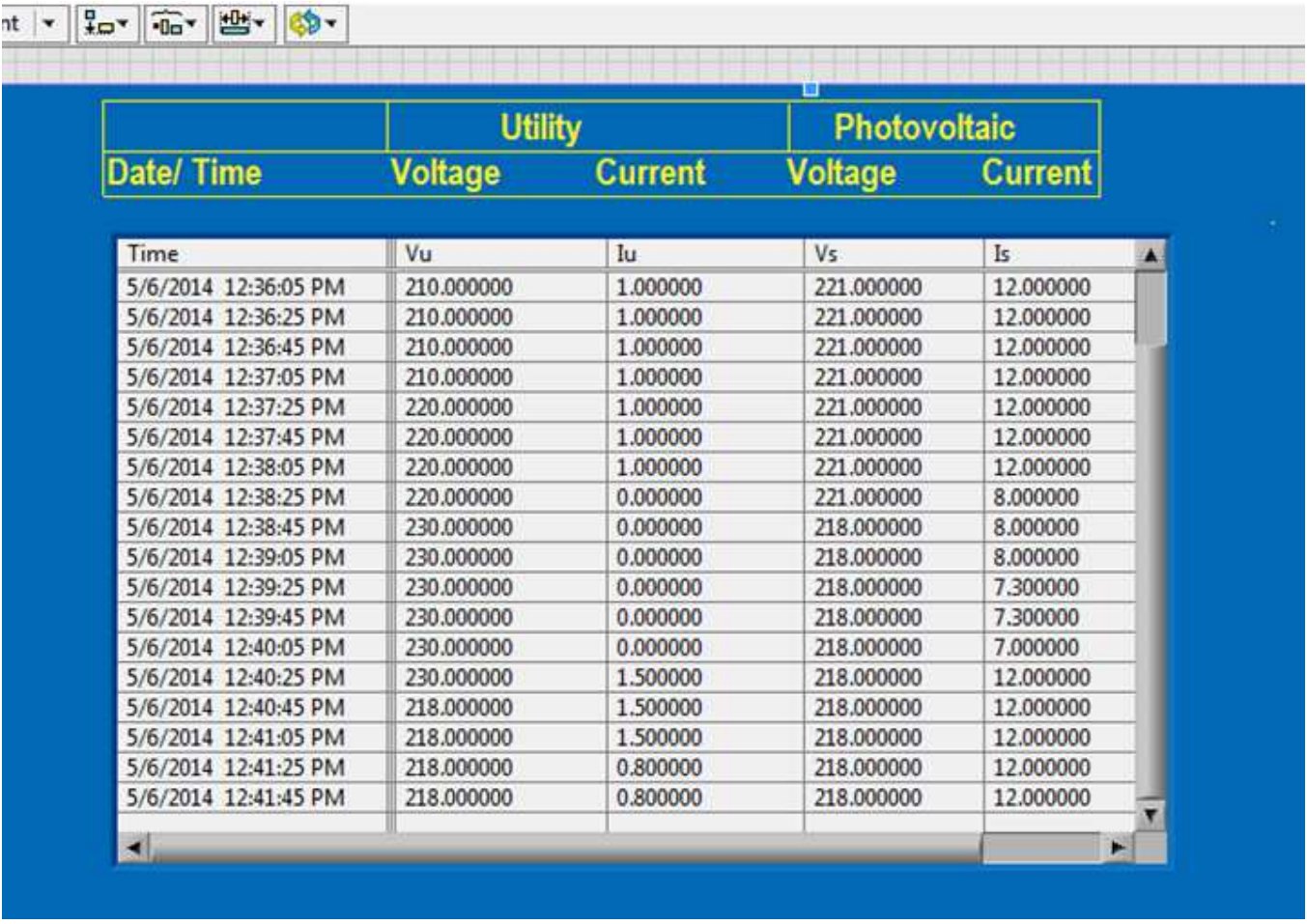

Figure 7. Data Logging Window of EMC.

\section{Experimental Result}

Proposed system is implemented on Spartan 3AN FPGA starter kit and Lab VIEW. Verilog HDL programming is used in Xilinx ISE to implement Smart Energy Management
Systems controller on FPGA. For hardware implementation FPGA, two CTs and two PTs are installed to gather information of photovoltaic power system and utility source. ZigBee pair is used to transfer the essential information between LABVIEW and Spartan 3AN FPGA Starter kit. Photovoltaic power system is installed with the help of six 
solar panels, a charge controller, four batteries and an inverter. Energy Management Center is used as graphical user interface for the monitoring and data logging of voltages and currents of photovoltaic power system and utility source. It is developed in LABVIEW to communicate with FPGA.

Three energy management techniques are tested: efficiency based technique, user based technique and normal load control technique. Experimentations for these techniques are performed over three sunny days and five different electrical loads are used. In user based technique and normal load control technique loads are given priorities for the switching of load transferring to photovoltaic power system and utility source. The results of the three techniques are shown in Figure8 and 9.

Figure 8 shows the power consumptions decrease from 7:00 to 13:00 in both user based and effeciency based techniques. In comparission with normal load control technique, effeciency based and user based techniques have improvement of 10.46 percent and 9.03 percent respectively in over all power consumption as shown in Figure 9. Above the dotted line utility source is used by the load. Effeciency can be further improved by extending battery backup and increase in number of photovoltaic pannels installation.

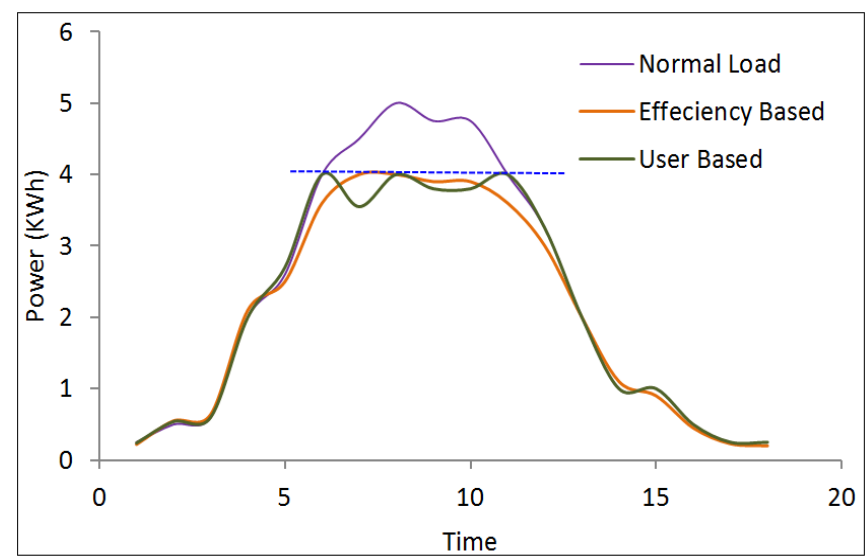

Figure 8. Power Consumption w.r.t time in SEMS.

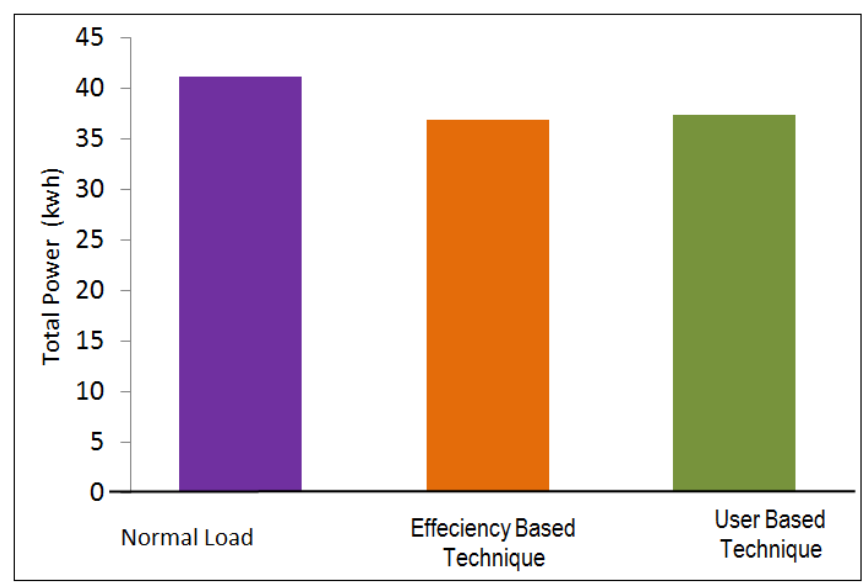

Figure 9. Experimental Result of Implemented SEMS.

\section{Conclusion}

The SEMS is designed for managing/improvement of energy efficiency of photovoltaic power system and utility source. The system is designed on Xilinx ISE using Spartan 3AN FPGA and LAB VIEW. The proposed system is cost effective which improves energy efficiency and gives an incentive to user. Users can easily monitor and manage their electricity loads according to the requirements by using Energy Management Center. Information of the daily consumed electricity is also stored in a file. User can also view their daily consumptions. If the number of photovoltaic panels and storage backup are improved, the efficiency of energy can be improved.

\section{References}

[1] Insung Hong, Byeongkwan Kang, and Sehyun Park "Design and Implementation of Intelligent Energy Distribution Management with Photovoltaic System" IEEE Transactions on Consumer Electronics, Vol. 58, No. 2, May 2012

[2] Saifur Rahman, Manisa Pipattanasomporn and Murat Kuzlu "An Algorithm for Intelligent Home Energy Management and Demand Response Analysis" IEEE Transactions on SMART GRID, Vol. 3, 2012, Page(s): 2166 - 2173

[3] Shaalan , Hesham E. “ Generation of Electric Power" Hand book of Electric power calculation,3rd ed. 2003, Mc-Graw-Hill.

[4] Kaplan, S. M. Smart Grid Electrical Power Transmission: Background and Policy Issues. The Capital.Net, Government Series. 2009 Page(s): 1-42

[5] F.Baig, A.Mahmood, N.Javaid, S.Razzaq, N.Khan and Z.Saleem "Smart Home Energy Management System for Monitoring and Scheduling of Home Appliances Using Zigbee" Journal of Basic and Applied Scientific Research, 2013, Page(s)880-891.

[6] Xunming Dengand, Eric A. Schiff "Amorphous Silicon-based Solar Cells" University of Toledo, Toledo, OH, USA, Syracuse University, Syracuse, NY, USA

[7] K. Touafek, M. Haddadi, and A. Malek, "Modeling and Experimental Validation of a New Hybrid Photovoltaic Thermal Collector," Energy Conversion, IEEE Transactions on, vol. 26, pp. 176-183, 2011.

[8] Xiaobin Huang, Beibei Jiang "Research on Lithium Battery Energy Storage System” IEEE Journals \& Magazines, 2011

[9] Anjali Singhal and R.P. Saxena "Software Models for Smart Grid" IEEE, SE-Smart Grids, Zurich, Switzerland, 2012, Page(s): $42-45$

[10] Frank Vahid "Digital Design with RTL Design, Verilog and VHDL" 2010. John Wiley and Sons. p. 247. ISBN 978-0-470-53108-2.

[11] Pradeep.P*, T.D.Sunil and M.Z.Kurian "Design of power management system using artificial intelligence based on FPGA" World Journal of Science and Technology 2012, 2(10):56-60 
[12] Xiaobin Huang, Beibei Jiang "Research on Lithium Battery Energy Storage System” IEEE Journals \& Magazines, 2011

[13] Zhang Guoju , Tang Xisheng, Qi zhiping "Research on Battery Supercapacitor Hybrid Storage and its application in MicroGrid” IEEE Journals \& Magazines, 2010.

[14] Kyle Thomas, V. A. Centeno, A. G. Phadke "Three-phase Instrument Transformer Calibration with Synchronized Phasor Measurements” IEEE Journals \& Magazines, 2011.

[15] James Momoh, SMART GRID Fundamentals of Design and Analysis, IEEE Press Series John Wiley \& Sons, 2012

[16] A Hajizadeh and MA Golkar. "Intelligent power management strategy of hybrid distributed generation system". International journal of Electrical Power and Energy System, 29(10), 2007.

[17] G Dawei, J Zhenhua, and L Qingchun. "Energy management strategy based on fuzzy logic for a fuel cell hybrid bus". Journal of Power Sources, 1(185), 2008.
[18] Abbas A. Akhil, Georgianne Huff, Aileen B. Currier, Benjamin C. Kaun, Dan M. Rastler, Stella Bingqing Chen, Andrew L. Cotter, Dale T. Bradshaw, and William D. Gauntlett, "DOE/EPRI 2013 Electricity Storage Handbook in Collaboration with NRECA" Sandia National Laboratories Albuquerque, New Mexico and Livermore, California, July 2013

[19] S Abras, S Ploix, S Pesty, and M Jacomino." A multi- agent design for a home automation system dedicated to power management". In Christos Boukis, Aristodemos Pnevmatikakis, and Lazaros Polymenakos, editors, AIAI, volume 247 of IFIP, pages 233-241, 2007.

[20] Clark W. Gellings, P.E. "The Smart Grid: Enabling Energy Efficiency and Demand Response" e Fairmont Press, Inc., 2009. 